\title{
Optimal Charge/Discharge Scheduling of Batteries in Microgrids of Prosumers
}

\author{
M. Ruiz-Cortés, E. González-Romera*, R. Amaral-Lopes, E. Romero-Cadaval, Senior Member, IEEE, \\ J. Martins, Senior Member, IEEE, M.I. Milanés-Montero and F. Barrero-González
}

\begin{abstract}
Integration of renewable energy sources, active role of consumers and energy management systems are currently among research priorities in energy systems. This paper proposes an innovative coordinated energy scheduling for a microgrid of neighbor prosumers with different consumption patterns. All prosumers have photovoltaic generation systems, Li-ion batteries as energy storage systems and regular household loads. A genetic algorithm is used to schedule each prosumer's battery charge/discharge, with the aim of reducing energy exchange losses by minimizing the power in the point of interconnection of the microgrid with the main grid, with several advantages compared to classical optimization objectives, and without worsening battery lifespan degradation. Individual and coordinated strategies are compared and self-consumption and self-sufficiency of the prosumers' set are evaluated with the aim of showing the advantage of coordination. The paper concludes that coordinated operation can contribute to improve the exploitation of energy resources in the prosumer microgrid, reducing the amount of energy interchanged with the distribution grid by approximately $13 \%$, and, at the same time, avoiding increasing the battery cycling and consequent degradation.
\end{abstract}

Index Terms - Battery management systems, Energy resources, Energy storage, Genetic algorithms, Microgrids, Selfconsumption.

\section{INTRODUCTION}

$\mathrm{N}$ OWADAYS, the interest of using renewable energy sources to improve self-consumption in residential distribution grids is generally accepted. Besides contributing to reduce greenhouse gas emissions, economic advantages arise for both consumers and grid operators [1]. The European Strategic Energy Technology (SET) Plan [2] establishes, among its key actions, taking European renewables sector into a world leading position, reinforcing consumers role in the energy systems and improving energy efficiency in general.

In a new scenario with distributed energy resources (DERs),

This work was supported by the Spanish Agencia Estatal de Investigación (AEI) and Fondo Europeo de Desarrollo Regional (FEDER), under Project TEC2016-77632-C3-1-R (AEI/FEDER, UE).

M. Ruiz-Cortés, E. González-Romera, E. Romero-Cadaval, M.I. MilanésMontero and F. Barrero-González are with the Electrical, Electronic and Control Engineering Department, University of Extremadura, Badajoz 06006 Spain. (e-mail: meruizc@peandes.es, evagzlez@unex.es, eromero@unex.es, milanes@unex.es, fbarrero@unex.es).

R. Amaral-Lopes and J. Martins are with the Electrical Engineering Department, NOVA University of Lisbon, Caparica 2829-516 Portugal. (email: rm.lopes@campus.fct.unl.pt, jfmartins@fct.unl.pt). two key concepts have emerged in research literature: prosumers and microgrids. A prosumer is defined as a combination of an energy producer and a consumer, i.e., it is a user able to both obtain power from the grid and deliver power to the grid [3]. Prosumers are owners of small end-user production installations, whose main goal refers to the optimization of energy usage according to economic goals, while maximizing their self-consumption and interacting bidirectionally with the distribution grid. On the other hand, microgrids are defined in literature as power systems which include loads and DERs (such as distributed generators, storage devices or controllable loads) that can be operated in a controlled and coordinated way either connected to the main grid or islanded [4]. They have been proposed as future Smart Grid structures [5]. Since a group of prosumers connected to the same utility feeder can be seen as DERs with loads, it can also be considered as a microgrid.

A classical prosumer energy management system (EMS) aims to achieve economic advantages by shaving power peaks, reducing energy purchase and by shifting demand according to generation availability and energy price [1]. Under this premise, individual prosumers are only concerned about their own benefit, and they neither support nor are supported by other prosumers. Energy storage systems (ESS) are key elements to achieve such objectives if the considered primary energy resources are intermittent, as it is the case of photovoltaic (PV) systems. Batteries, and particularly Li-ion based, are among the most widely used for ESS at the residential sector. Therefore, combination of photovoltaic generation systems and batteries are commercially available for prosumers to improve their self-consumption and selfsufficiency [6].

However, single operation may prevent the user to obtain the previously mentioned advantages if either its storage device capacity or state of charge $(\mathrm{SoC})$ level is not adequate. This issue could be solved by cooperative operation. Prosumer EMSs are rarely coordinated with other prosumers or grid operators to optimize the global performance of a community or microgrid, or to achieve common goals. Microgrid concept encourages coordination [7], since joint energy management can have economic advantages when the microgrid is gridconnected and it is essential in isolated operation. Previous works [3], [8] developed the concept of "peer-to-peer (P2P)" supply, evaluating its impact on the self-consumption of a community of prosumers. Although some of them consider the 
presence of $\mathrm{PV}$ generation and storage, in these works $\mathrm{P} 2 \mathrm{P}$ supply is provided only by the generation surplus, without the involvement of stored energy. On the other hand, several works have started to study the design of microgrid markets. For instance, an internal price model is articulated in [8], whereas [9] claims the aggregation of small prosumers to take part into energy markets in a more efficient way. However, [10] concludes that more research is still required to determine suitable socio-economic incentives of participants in the market.

In this paper, a novel scheduling method for the battery charge and discharge of a group of prosumers in a microgrid is proposed. Each prosumer's installation consists of a PV generation plant, a Li-ion battery based ESS and several household loads, whose operation is studied, both, from the individual viewpoint and also from the coordinated perspective as a microgrid.

Genetic algorithms (GA) are used to optimally match generation, load and storage in both individual and coordinated scenarios, based on one-hour resolution forecasted generation and load profiles. GA have been widely used in literature with energy optimization purposes in microgrids. However, they are commonly focused on economic objectives [1], [11], [12] or prosumers' optimal clustering [9], [13] instead of optimizing the exploitation of the microgrid DERs.

In this work, power sharing among batteries is scheduled, with the aim of minimizing the power imbalance between forecasted generation and load and planned storage, during the 24 hours of a one-day time horizon. That is to say, power in the point of interconnection between the microgrid and the main grid is targeted to be minimized to reduce energy exchange losses, both during energy import and export. This objective function presents advantages compared to classical objective functions, like peak shaving, economical profits or self-consumption. As optimization model constraints, adequate SoC range is assured for every battery, charge/discharge power is limited to converter capacity and power gradient between consecutive hours is also restricted to reduce battery degradation [14].

The novelty of this work lies in two main contributions: firstly, the design of a coordinated strategy for prosumers to integrate them into a microgrid concept, which shows clear advantages compared to individual operation; secondly, a novel optimization function is defined for GA. It is not focused on maximizing profits or reducing costs, but on reducing energy exchange losses by minimizing power in the point of interconnection. Therefore, conclusions are not dependent on distinct country energy prices and feed-in tariff schemes. As it is discussed along the paper, this objective function gathers advantages of several usual optimization targets.

The rest of the paper is organized as follows. In Section II, the prosumer microgrid assumed as case study is detailed. Section III describes the GA algorithm designed for optimization. Section IV defines the evaluation indices. Section V presents and discusses results. Finally, conclusions are presented in Section VI.

\section{CASE StUdy}

The system analyzed in this paper consists of a low voltage grid with $N$ residential end users, indexed by $i$. These homes show an energy demand that results from the operation of typical household electrical devices, such as lighting, air conditioning, television sets, heating or ventilation systems (if they are applicable), just to name a few. Each user $i$ is equipped with a PV array and a battery as ESS, with the aim of reducing the resulting grid interaction, both in terms of power and energy, and consequently achieving economic savings in their electricity bills. In this way, the $N$ users are consumers and producers, so they become $N$ interconnected prosumers.

Here, it is assumed that all prosumers belong to the same residential area, so they are located close to each other. Nowadays, developers usually preinstall home facilities (including energy resources) at the building construction phase, when occupancy and energy requirements of each household is not always known, and it is common that dwellings of a newly-constructed building promotion are equipped with identical devices. Following this trend, it has been supposed that all prosumers of the studied example system have the same installed peak PV - rated at $4 \mathrm{~kW}-$ power and identical ESSs. Each one of the modelled ESSs consists of a Li-ion battery with high nominal capacity of 6 $\mathrm{kWh}$, connected to a power converter that limits the battery output power to a maximum amount of $2 \mathrm{~kW}$ (+ charging, discharging). Their contracted power is equal to $4.6 \mathrm{~kW}$, according to current normalized power levels offered in Spain for single-phase medium-load installations. For this specific study, $N=2$ is chosen for the simulation model, as in [15]. Fig. 1 depicts the conceptual architecture of the modelled system with relevant data used in the simulation. Battery nominal voltage and maximum current values follow the specifications of the commercially available Li-ion NMC battery BMZ ESS 7.0 [16].

Five different scenarios have been assessed to establish which operation mode is the most efficient when an optimal charge/discharge scheduling is applied. It has been assumed that prosumers produce the same PV generation working at the Maximum Power Point (MPP). As they are located in near places, it is presumed that they receive very similar solar irradiance [3]. However, their electrical consumption in a certain day has not to be equal to a neighbor's demand as a consequence of their individual occupancy or electric

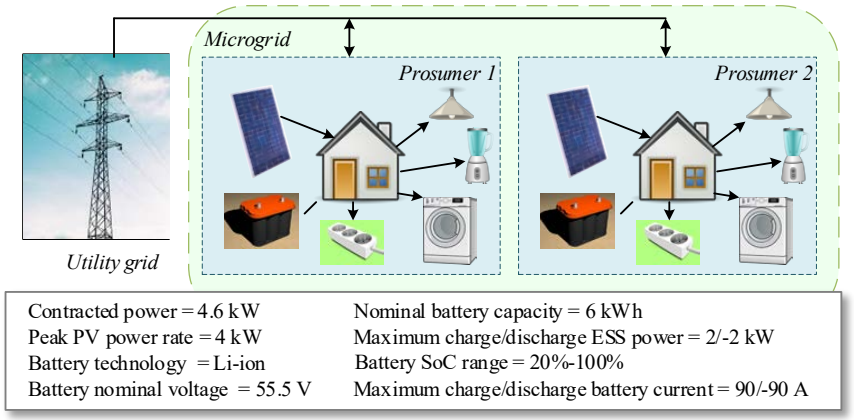

Fig. 1. Conceptual architecture of the simulation system. 
consumption patterns. For this reason, each scenario considers a different combination of possible load behaviors for both prosumers of the simulation system. The PV power generated by each prosumer (based on climate data from a nearby weather station) and the different demand profiles used in each scenario (measured from real households) as input data are shown in Fig. 2. In this paper, curves in Fig. 2 are assumed to be forecasted profiles, with one-hour resolution in this case, for a particular day. Table I summarizes the proposed scenarios and the corresponding demand profiles. The terms "Net-zero Balance", "Demand Surplus" and "Generation Surplus" are used when the daily energy import from the grid is equal, higher or lower, respectively, than the amount of energy exported.

Furthermore, this diversity of scenarios provides the chance to evaluate the proposed strategy in case of different PV system sizes. For example, scenario 4 could also be valid for the case of two prosumers with similar electricity consumption but different generation capacity (prosumer 1, characterized by "demand surplus", would have a smaller PV array size than prosumer 2). The opposite case could be seen in scenario 5, where prosumer 1 would present "generation surplus" as a result of a higher PV array size than prosumer 2.

\section{DAY-AHEAD SCHEDULING OF BATTERIES WITH GENETIC ALGORITHM}

\section{A. Problem formulation}

In the literature related to battery energy management, most approaches simply charge the battery up to max capacity when local generation is higher than load, and discharge it up to min capacity in the opposite situation, as is the case of [17].

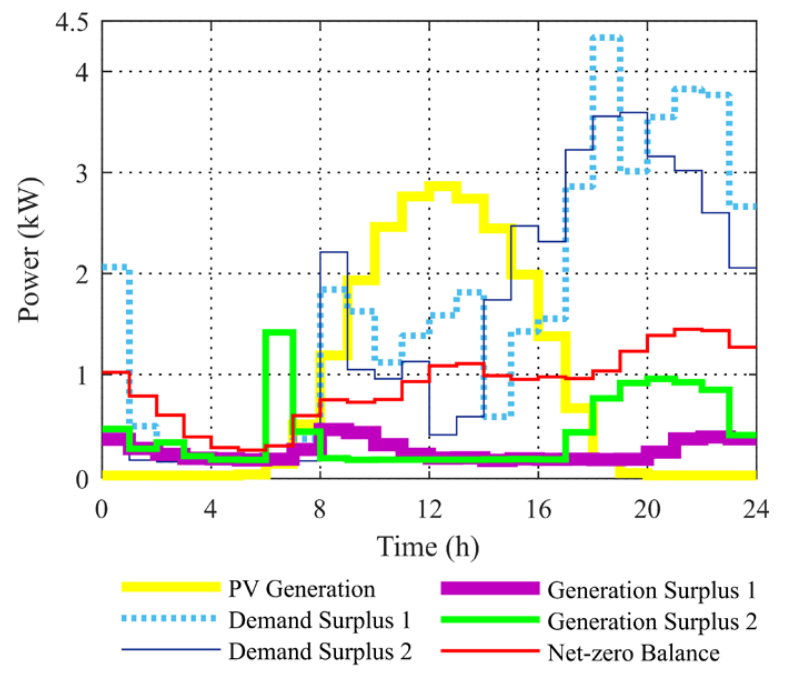

Fig. 2. Generation and demand power profiles used for the analyzed scenarios.

TABLE I

ANALYZED SCENARIOS AND DEMAND PROFILES USED IN EACH ONE

\begin{tabular}{c|c|c}
\hline \hline Scenario & Prosumer 1 & Prosumer 2 \\
\hline 1 & Demand Surplus 1 & Generation Surplus 1 \\
\hline 2 & Demand Surplus 1 & Demand Surplus 2 \\
\hline 3 & Generation Surplus 2 & Generation Surplus 1 \\
\hline 4 & Demand Surplus 1 & Net-zero Balance \\
\hline 5 & Generation Surplus 2 & Net-zero Balance \\
\hline \hline
\end{tabular}

However, there are more dispatch possibilities, such as planning it according to specific characteristics and stakeholders' goals [18]. In contrast, the present paper proposes a novel technique based on GA to schedule a dayahead battery operation, with the aim of minimizing the power in the point of interconnection with the main grid and consequent energy exchange losses.

The battery charging/discharging procedure is programmed for each hour of the next day taking into account forecasted generation and load profiles, which are usually known with enough accuracy 24 hours before. One-hour resolution has been used because it is the most convenient frame to get proper predictions addressing generation and demand. Moreover, electricity market managers, such as OMIE in the Spanish-Portuguese joint market, work in that way, programing energy dispatch for the 24 hours of a one-day time horizon based on generation and load forecasted the previous day. For this reason, electricity markets usually provide hour by hour prices, so end users can plan their energy sources with this time interval if an economic goal is pursued.

It is worth noticing that the objective of this paper focuses on planning the average power of the battery for each hour of the day in advance. Real time adjustments are required over this hourly setpoints, to handle forecasting inaccuracy and instantaneous changes. It is widely assumed that microgrid control is commonly performed following a hierarchical scheme [4]. A primary quick control solves real-time power balance to keep stable frequency and voltage and a secondary slower control performs energy management system. In this paper, setpoints for batteries generated by secondary control are the target results.

The scheduling algorithm is valid for both individual and coordinated operation modes. In the first one, each battery is programmed independently, whereas in the second one, all DERs of the microgrid are considered to be a single unit and power sharing between batteries is carried out.

Thus, each prosumer $i=1,2, \ldots, N$ is characterized by its demand and generation forecasted active power curves. Generation $\mathbf{G}$ and demand $\mathbf{D}$ may be expressed mathematically as vectors with as many elements as time slots, $\tau$, of a determined duration, $\Delta t$, one desires to schedule, i.e. $\mathbf{G}=[G(1), G(2), \ldots, G(\tau)]$ and $\mathbf{D}=[D(1), D(2), \ldots, D(\tau)]$. In this study, a full day horizon with time intervals of one hour is considered, i.e. $\tau=24$ time slots. When the individual operation mode is applied for prosumer $i, \mathbf{G}$ and $\mathbf{D}$ are its corresponding generation and demand data $\mathbf{G}^{\mathbf{i}}$ and $\mathbf{D}^{\mathbf{i}}$, whereas in the coordinated operation mode aggregated generation and demand data are used instead. The aggregated generation and demand are calculated as the sum of the $N$ considered prosumers' individual generation and load, respectively, as indicated in (1) and (2).

$$
\begin{aligned}
& \mathbf{G}=\sum_{i=1}^{N} \mathbf{G}^{\mathbf{i}} \\
& \mathbf{D}=\sum_{i=1}^{N} \mathbf{D}^{\mathbf{i}}
\end{aligned}
$$


The dispatched active power schedule of each prosumer's battery $i, \mathbf{P}_{\mathrm{B}}^{\mathrm{i}}$, may also be written as a vector of $\tau$ elements. Each element represents the power stored or delivered by the battery for every time slot and can present either positive or negative values depending on the direction of the power flow ( + charging and - discharging). All $\mathbf{P}_{\mathrm{B}}^{\mathrm{i}}$ contribute to modify the original demand $\mathbf{D}$ as shown in (3)

$$
\mathbf{D}_{\text {mod }}\left(\mathbf{P}_{\mathbf{B}}^{\mathbf{i}}\right)=\mathbf{D}+\sum_{i=1}^{N} \mathbf{P}_{\mathbf{B}}^{i}
$$

where $\mathbf{D}_{\text {mod }}\left(\mathbf{P}_{\mathbf{B}}^{\mathbf{i}}\right)$ is the modified demand, i.e., original demand plus battery power. Note that in individual operation mode, $N$ $=1$. In this study, since they do not significantly influence the comparison of strategies, batteries related power losses have been neglected. Regarding the PV inverter, a 90\% efficiency has been considered.

\section{B. Objective Function}

The problem consists of finding appropriate values for every $\mathbf{P}_{\mathbf{B}}^{\mathbf{i}}$ vector which assure that specific targets are achieved. In this study, the pursued objective is minimizing the mismatch between forecasted PV generation and demand for a 24-hour time horizon when batteries are used, i.e., the difference between generation $\mathbf{G}$ and modified demand $\mathbf{D}_{\text {mod }}\left(\mathbf{P}_{\mathbf{B}}^{\mathbf{i}}\right)$ at every time interval $t=1,2, \ldots, \tau$. This objective can be described by (4), where the square operator is applied to penalize the highest errors.

$$
\operatorname{minimize} f_{\text {mismatch }}(t)=\left[D_{\text {mod }}(t)-G(t)\right]^{2}
$$

This objective function, also called fitness function, has been chosen instead of more usual optimization targets, due to several reasons:

- The most usual optimization target is prosumer economic profit. Most works aim at minimum energy costs [19], [20], proposing a cost function for the generated and stored energy and an example price for the purchased and sold energy. However, the objective function is strongly dependent on the different energy policies carried out by countries. As [10] argues, "legislative rules determine how taxes and fees are distributed and in which way the market is integrated into the traditional energy market and energy supply system". Therefore, governments are responsible of deciding either they support new electricity market models or prefer holding the traditional energy system. A comparison among feed-in tariff or net metering schemes for self-consumption in different countries in the European Union is shown in [21]. According to [21], remuneration for surplus electricity sold to the grid ranges from no compensation (Slovakia) to feed-in tariffs (Denmark) or market price plus management premium (Germany). In addition, grid and system cost contribution is exempted (United Kingdom) or allocated (Cyprus), depending on the country. Besides, self-consumption policies suffer frequent changes (for example, Spain and France sanctioned their rules later). In this way, any study based on economic prices will be subjected to a particular regulation framework, and its results will not be applicable to a different or general scenario.

- Another classical objective function is related to peak shaving. It is advantageous for distribution system operators (since it reduces the grid capacity requirement) and for consumer (who reduces the energy purchased at peak price hours) [22]. In a bidirectional generation-load system, peak shaving makes sense to be applied in both peak periods (max demand/energy import and max generation/energy export) [23]. The proposed objective function aims to apply peak shaving at both peak periods and also at every hour, to simultaneously reduce the amount of energy to be purchased or sold.

- A new objective is added related to microgrids. It is well known that microgrids must be able to work either isolated or connected to the main grid. This objective function allows a smooth transition between both states, as it reduces the interconnection power at every hour.

Additionally, the optimal generation-load matching advocated by the objective function implies various consequences (related to smart grid targets):

1) Energy demand reduction from the grid's point of view;

2) Higher autonomy of end users regarding the grid;

3) Lower energy flows between end users and grid;

4) Power losses decrease;

5) Improved integration of renewable energy sources;

6) Emissions reduction and sustainability increase;

7) Lower grid congestion risk;

8) Lower end users' vulnerability to grid disturbances;

9) Electrical bidding reduction.

\section{Problem Constraints}

The considered approach pursues not only optimal operation of the batteries, but also battery degradation avoidance. Investment, installation and replacement costs of batteries represent a large percentage of the total cost of the prosumers' energy system. Therefore, battery lifespan preservation is a key issue to be considered when operational strategies are designed [15].

This is the motivation for including a set of constraints which any solution $\mathbf{P}_{\mathbf{B}}^{\mathbf{i}}$ of the objective function must comply with, as detailed below:

1) Safe operational SoC range

Aging due to battery cycling is strongly conditioned by deep discharges [15], [24], and high deterioration may be caused by overcharge situations [15], [25]. For this reason, it is important that batteries work within a safe operational SoC range, i.e. $S o C_{\text {min }}^{i} \leq S o C^{i}(t) \leq S o C_{\text {max }}^{i}$, where $S o C_{\text {min }}^{i}$ and $S o C_{\max }^{i}$ are the minimum and the maximum SoC levels allowed for battery $i$, respectively. The limits' values must be properly established by the technology of the battery and the selected operation [26]. In the present 
case study, $S o C_{\min }^{i}=20 \%$ and $S o C_{\max }^{i}=100 \%$ for Li-ion batteries were chosen, taking [27] as a reference. Thus, each value of $\mathbf{P}_{\mathbf{B}}^{\mathbf{i}}$ has to verify constraint (5)

$$
S o C_{\text {min }}^{i} \leq \sum_{t=1}^{\tau}\left[P_{B}^{i}(t) \Delta t\right] / C_{\text {nom }}^{i}+S o C_{\text {init }}^{i} \leq S o C_{\text {max }}^{i},
$$

where $C_{n o m}^{i}$ is the nominal capacity of every battery and $S o C_{\text {init }}^{i}$ is their corresponding SoC at starting time, to assure the $\mathrm{SoC}$ at each $t$ is bounded in the considered range. Herein, initial SoC values have been selected different for each battery, using $S o C_{\text {init }}^{1}=83 \%$ and $S o C_{\text {init }}^{2}$ $=50 \%$. As mentioned before, $C_{\text {nom }}^{1}=C_{\text {nom }}^{2}=6000 \mathrm{Wh}$, and the considered duration time interval $\Delta t=1 \mathrm{~h}$.

2) Charge/Discharge Power Limitation

A constraint related to the power level supplied by ESS at every time slot must also be considered to avoid possible damages. Equation (6) expresses the allowed power range

$$
P_{\max , \text { dis }}^{i} \leq P_{B}^{i}(t) \leq P_{\max , c h}^{i},
$$

where $P_{\text {max,dis }}^{i}$ and $P_{\text {max, } c h}^{i}$ are the maximum power that can be provided or absorbed by the ESS $i$. Notice that this power limitation do not necessary correspond to battery specifications, since in most cases it is due to the interfaced power converter characteristics [17]. In the present case study, it is assumed that $P_{m a x, d i s}^{i}=-2000 \mathrm{~W}$ and $P_{\text {max }, c h}^{i}=2000 \mathrm{~W}$, according to the selected converter capacity.

3) Power Gradient Restriction

Power gradient between consecutive hours is also restricted to reduce battery degradation. Large swings in SoC during cycling cause accelerated battery degradation [28]. Additionally, alternating charging, discharging, and idle time segments take part of the cyclic aging model presented in [24]. Equation (7) describes this constraint, which aims to ensure a stable battery behavior without large power oscillations between two consecutive time intervals.

$$
\left|P_{B}^{i}(t)-P_{B}^{i}(t+1)\right| \leq \Delta P_{B}
$$

The maximum allowed power gradient, $\Delta P_{B}$, has been quantified in $300 \mathrm{~W}$ for this case study. In the measured demand time series used as input data, once the daily demand profile was averaged for a year, the highest difference observed between consecutive hours in this average profile is $300 \mathrm{~W}$.

\section{Algorithm implementation}

GA was selected for solving the optimization problem due to its well-known suitability for combinatorial optimization problems with large search space [29], which results from its higher ability to search the solution in the entire space and to avoid local-optima than conventional optimization techniques [12].

GA emulates natural selection process. It begins by creating a random initial population from the solution space which is a first set of possible solutions. Each possible solution is expressed as a vector called chromosome and composed by a required number of elements (or genes) to represent the attributes of each individual. Similarly to evolutionary theories, a possible solution is able to take part in a new population creation depending on its fitness for the objective function. Thus, GA creates a sequence of new populations (children) using the individuals of the current generation (parents) according to their fitness value. In each generation, GA tries to find the best possible solution until one stopping criteria is met.

In the present case study, GA has been employed to find a chromosome of $N \cdot \tau$ genes (the number of variables involved in the optimization problem), denoted by $\mathbf{P}_{\mathbf{B}} . \mathbf{P}_{\mathbf{B}}$ is a row vector composed by the $N$ concatenated strings $\mathbf{P}_{\mathbf{B}}^{\mathbf{i}}$, which are the intended solutions. Thus, if the individual operation mode is used, $\mathbf{P}_{\mathbf{B}}$ has $N \cdot \tau=1 \cdot 24=24$ elements, where each one represents the scheduled power for the corresponding onehour time slot. In contrast, when the coordinated operation is applied, $N \cdot \tau=2 \cdot 24=48$ and $\mathbf{P}_{\mathbf{B}}$ has 48 elements, the first 24 elements correspond to the battery scheduling of prosumer 1 and the last 24 to the battery scheduling of prosumer 2 .

Aiming to assure that the initial population satisfies (6), the initial search space is narrowed down to the range [-2000, 2000]. Additionally, to deal with the random behavior of initial population and unforeseeable nature of creation of new ones, a series of iterations which warrants a solution with proper quality was carried out. Every iteration uses the best solution founded in the previous iteration as initial population, so fitness values only can improve iteration by iteration. A number of 10 iterations was selected in this case to reach a compromise between time execution and solution quality.

Regarding constraints, the charge/discharge power limitation is expressed as a lower (discharge) and an upper (charge) bounds, whereas the safe operational SoC range and power gradient restrictions are treated as inequality constraints. Constraint tolerance was set at 0.001 to determine the feasibility of the solution proposed by the GA with respect to constraints. The Augmented Lagrangian Genetic Algorithm (ALGA) constraint solver provided by MATLAB ${ }^{\circledR}$ was used to handle constraints [30]. It is a penalty-based method, which punishes unfeasible solutions with respect to constraints, minimizing with GA a sequence of subproblems that combine the fitness function and nonlinear constraint functions. Advantages offered by this approach are explained in [31] and [32].

\section{EVALUATION INDICES}

The performance of the individual and coordinated operation modes with the proposed scheduling of batteries has been evaluated in each scenario using a set of indicators extensively accepted in the literature. All of them can be obtained for different periods of time, comprised between $t_{1}$ and $t_{2}$. Here, the analysis period has been particularized to a full day with one-hour resolution, so $t_{1}=1$ and $t_{2}=\tau$.

Equation (8) calculates the load instantly matched $(L M)$ by the local production with the scheduling of batteries, delimited by the applicable PV generation and modified demand. 


$$
L M(t)=\min \left[D_{\text {mod }}(t), G(t)\right]
$$

Using $L M$, self-consumption and self-sufficiency rates can be defined as (9) and (10), respectively.

$$
\begin{aligned}
& S C=\sum_{t_{1}=1}^{t_{2}=\tau} L M(t) / \sum_{t_{1}=1}^{t_{2}=\tau} G(t) \\
& S S=\sum_{t_{1}=1}^{t_{2}=\tau} L M(t) / \sum_{t_{1}=1}^{t_{2}=\tau} D(t)
\end{aligned}
$$

The self-consumption rate, denoted by $S C$, indicates the percentage of on-site generation that is instantly consumed by the prosumer/microgrid, whereas the self-sufficiency rate, $S S$, describes the percentage of demand instantly satisfied by the on-site generation [17], [26], [29]. In both cases, the electrical demand is considered once it has been modified by the scheduling of batteries.

In addition, the power interchange between the prosumer/microgrid and the distribution grid at every time slot, $P_{\text {grid }}(t)$, whose absolute value is targeted to be minimized, has been obtained using (11).

$$
P_{\text {grid }}(t)=G(t)-D_{\text {mod }}(t)
$$

Note that $P_{\text {grid }}(t)$ presents negative values when the local generation is lower than the modified demand and positive values in the opposite case. In the first case, the prosumer/microgrid imports energy from the distribution grid and exports energy to it in the second one.

Equation (12) represents the accumulated energy net balance, $E_{n e t}$, for the considered period of analysis.

$$
E_{n e t}=\sum_{t_{1}=1}^{t_{2}=\tau} P_{\text {grid }}(t)
$$

Accumulated energy export and import for the considered period of analysis have been calculated using (13) and (14) respectively.

$$
\begin{gathered}
E_{\text {exp }}=\sum_{t_{1}=1}^{t_{2}=\tau} P_{\text {grid }}(t) \forall P_{\text {grid }}(t)>0 \\
E_{\text {imp }}=\sum_{t_{1}=1}^{t_{2}=\tau} P_{\text {grid }}(t) \forall P_{\text {grid }}(t)<0
\end{gathered}
$$

Finally, the total amount of energy interchanged between the prosumer/microgrid and the main grid, $E_{\text {int }}$, has been assessed with (15) for the period of analysis, computing energy exports and imports in absolute value.

$$
E_{\text {int }}=\sum_{t_{1}=1}^{t_{2}=\tau}\left|P_{\text {grid }}(t)\right|
$$

\section{RESUlTS AND DISCUSSION}

The optimal scheduling of batteries has been validated through simulation using MATLAB ${ }^{\circledR}$ software tool. It has been applied to the system described in Section II for the five proposed scenarios in individual and coordinated operation modes. This section presents and discusses the main results obtained. For the sake of readability, some figures refer only to results obtained for scenario 1 , which has been considered the most interesting case.

Fig. 3 shows the results obtained regarding load matching in scenario 1 . With the individual operation, the scheduling of batteries achieves that prosumer 1 partially supplies its load at the end of the programmed day with the energy stored at noon in its battery. Prosumer 2 becomes self-sufficient, because it is able to supply its consumption with the energy stored in its battery at early morning. The battery is completely charged at noon and supplies the requested demand at evening, being still charged up to $70 \%$ at the end of the day (see Fig. 4(a)). Fig. 3(a) and Fig. 3(b) demonstrate that the scheduling complies with (6), because the difference between original and modified demands is lower than $2 \mathrm{~kW}$. Fig. 3(c) compares the global results obtained for the combined prosumers (prosumer $1+$ prosumer 2) when individual and coordinated operation modes are applied. It can be observed that the load matching is better with the coordinated operation, because the modified demand is higher at midday and lower at late evening than with the individual operation, so it allows using a higher amount of energy from the prosumers' DERs. Notice that modified demand is negative during some hours at early morning. It means that batteries provide more energy than consumption requires, so they directly deliver energy to the main grid. This does not clash with the objective function, because batteries are slightly discharged to increase the PV power utilization. In addition, limitations related to bidirectional exchanges between batteries and the main grid at specific hours of the

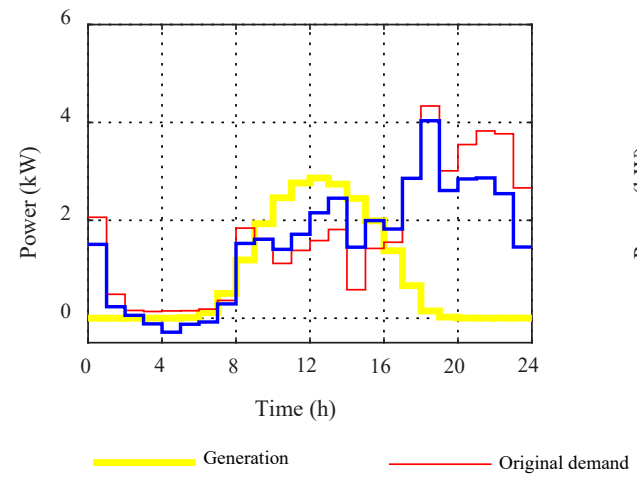

(a)

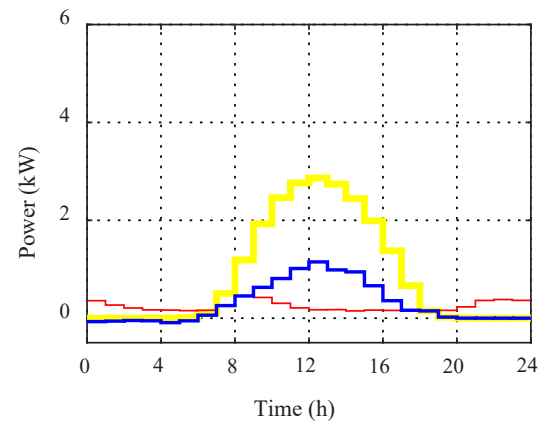

(b)

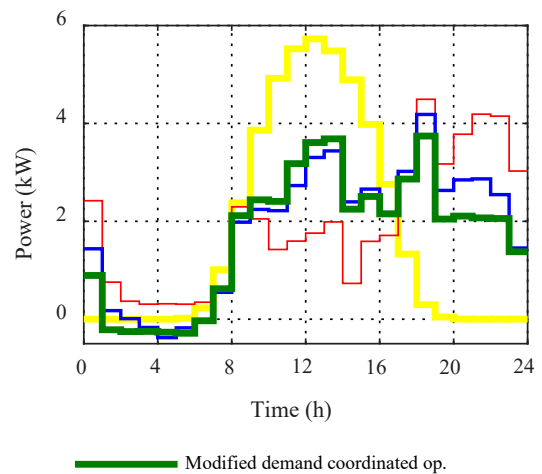

(c)

Fig. 3. Generation, original demand and modified demand profiles for (a) prosumer 1 individual operation, (b) prosumer 2 individual operation, and (c) global (prosumer $1+$ prosumer 2) individual and coordinated operation in scenario 1. 


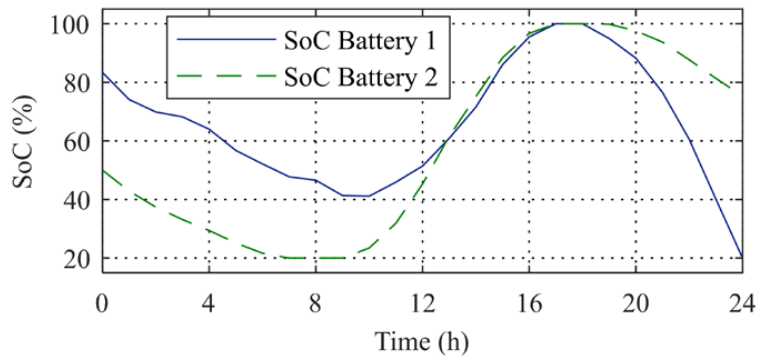

(a)

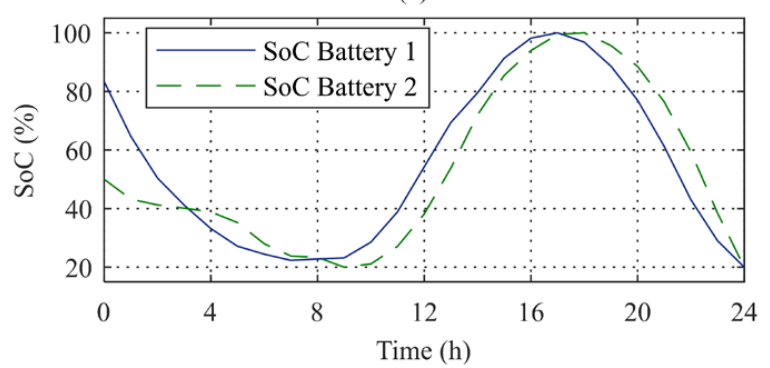

(b)

Fig. 4. Daily evolution of battery SoC with (a) individual operation, and (b) coordinated operation in scenario 1 .

day are not included among the formulated problem constraints.

Fig. 4 shows the SoC evolution of both batteries of the system during the planned full day in either operation mode for scenario 1 . The optimal day-ahead scheduling provides a smooth behavior of the batteries for both operation modes. In both cases, the same number of charge/discharge stages is required, which is related to battery lifetime, because frequency of use is one of the main factors of aging that govern degradation [28]. The smooth SoC evolution means that the gradient constraint is satisfied and that an oscillating behavior, characterized by damaging sharp power variations and unnecessary charge/discharge cycles, is avoided. In addition, the safety SoC limits are met. Although changes in $\mathrm{SoC}$ (charging/discharging power) are higher in cooperative mode during some periods, this power and changes between consecutive hours are limited by the problem restrictions. These constraints, in terms of current (nominal voltage has been assumed for the battery in this calculation), result in a maximum current of $19.5 \mathrm{~A}$ and a maximum current gradient between hours of $5.4 \mathrm{~A}$. Fig. 5 illustrates how these values are far from limits stated by manufacturer (Fig 1, $i_{B, \text { max charge }}$ and $i_{B, \max }$ discharge in Fig. 5). According to [33], the main concern of using lithium batteries at high currents is during charging, since manufacturers impose limits on the charging current that imply much smaller charging powers (3C) than discharging ones (between $8 \mathrm{C}$ and $15 \mathrm{C}$, the value of $\mathrm{C}$ means the relation between power and energy of the battery, that is, 4C would mean for a $1 \mathrm{kWh}$ battery to use it at $4 \mathrm{~kW}$ of power). In the case of this paper, values below $0,33 \mathrm{C}$ are always obtained due to constraints. Comparing both operation modes, it can be observed how the battery of prosumer 1 is not fully discharged in the individual operation (Fig. 4(a)) in spite of being a deficit scenario; however, when the coordinated operation is applied (Fig. 4(b)), a better use of the capacity of both batteries is

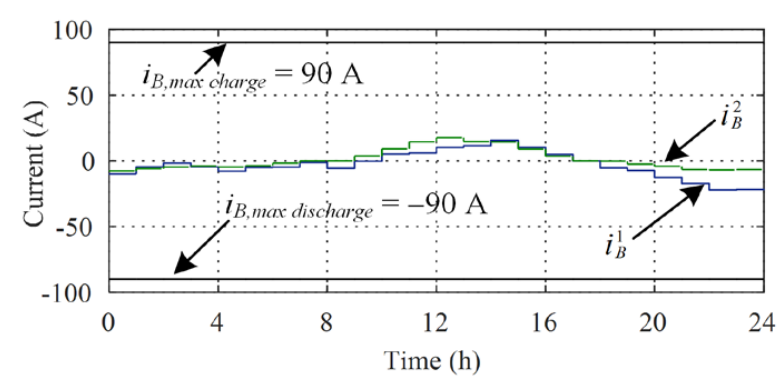

(a)

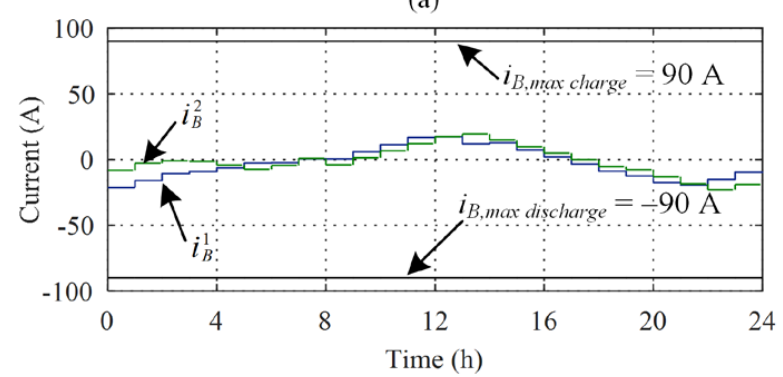

(b)

Fig. 5. Comparison of battery charge/discharge currents with individual operation (a) and coordinated operation (b) with respect to maximum current rates in scenario 1 .

achieved. Moreover, the resultant power sharing between batteries in the coordinated operation gets that they work in a more balanced way: at the beginning of the simulation, the battery of prosumer 1, which has a higher SoC than the other one, provides more energy until both batteries reach a close $\mathrm{SoC}$ value; since then, both batteries work together storing/delivering similar amounts of energy, as we can deduct from the parallel slope of both SoC curves. This behavior is reasonable taking into account that, in this case, both batteries present the same characteristics. It is beneficial for avoiding overutilization of one battery with respect to the other one and for reducing circulating currents, because it favors that batteries are all charging or all discharging [34].

The previous results allow us to conclude that the coordinated operation is more efficient than the individual operation, as simultaneous improvement of both $S C$ and $S S$ indices illustrates (see Table II). In scenario 1, the global $S C$ with coordinated operation is equal to $59.68 \%$ against $54.58 \%$ with individual operation, and the global $S S$ is also higher with the first one (54.60\% vs. $49.93 \%)$. Global $S C$ in individual operation has been calculated as the ratio between the TABLE II

$S C$ AND SS FOR THE DIFFERENT SCENARIOS AND OPERATION MODES

\begin{tabular}{c|c|c|c|c|c}
\hline \hline \multirow{2}{*}{ Scenario } & \multicolumn{3}{|c|}{ Individual Operation } & \multirow{2}{*}{$\begin{array}{c}\text { Coordinated } \\
\text { Operation }\end{array}$} \\
\cline { 3 - 5 } & & Prosumer 1 & Prosumer 2 & Global & 0.5968 \\
\multirow{2}{*}{1} & $S C$ & 0.7478 & 0.3437 & 0.5458 & 0.5460 \\
\hline \multirow{2}{*}{2} & $S S$ & 0.3908 & 1.262 & 0.4993 & 0.7696 \\
& $S C$ & 0.7478 & 0.7156 & 0.7317 & 0.4293 \\
\hline \multirow{2}{*}{3} & $S S$ & 0.3908 & 0.4281 & 0.4082 & 0.3116 \\
& $S C$ & 0.2678 & 0.3437 & 0.3058 & 0.8342 \\
\hline \multirow{2}{*}{4} & $S S$ & 0.5641 & 1.2620 & 0.8185 & 0.7802 \\
& $S C$ & 0.7478 & 0.6778 & 0.7128 & 0.5356 \\
\hline \multirow{2}{*}{5} & $S S$ & 0.3908 & 0.6778 & 0.4893 & 0.5145 \\
& $S C$ & 0.2678 & 0.6778 & 0.4728 & 0.6977 \\
\hline \hline
\end{tabular}

SS>1 means that the prosumer/microgrid is able to supply a higher load than it demands. 


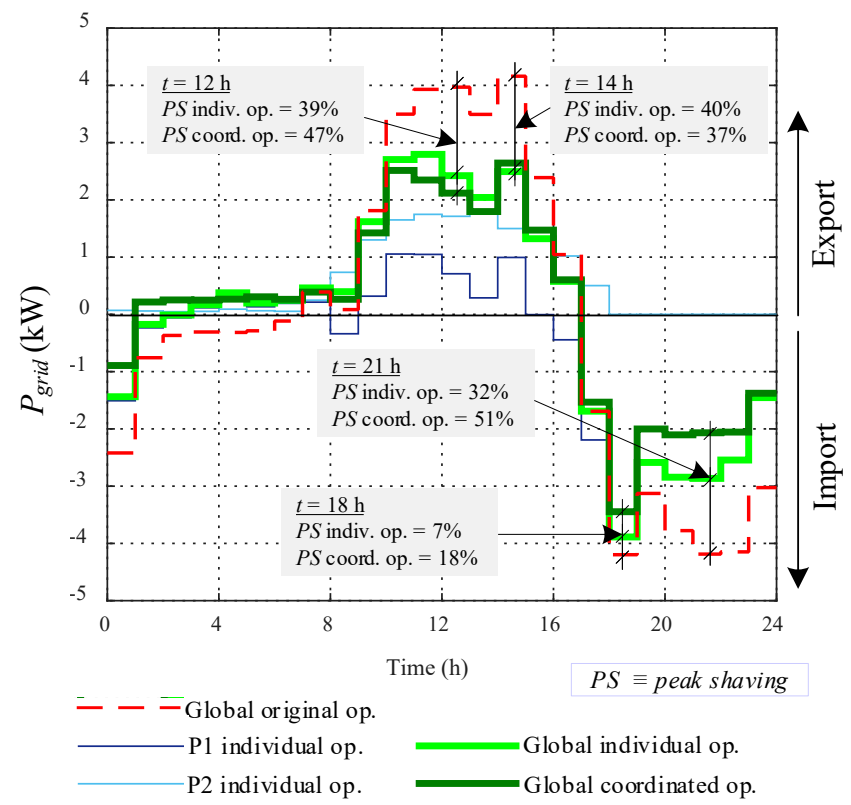

Fig. 6. Individual and global power net balance profiles for the different operations in scenario 1 .

aggregated load matched by the $N$ prosumers for the analyzed period and the aggregated generation of all of them in that period, whereas global $S S$ uses the aggregated demand for that period instead of the aggregated generation. According to [17], SS should not exceed $80 \%$ without excessively oversizing the system. The better performance of the scheduling of batteries in the coordinated operation mode is confirmed by the results obtained in the other four scenarios, detailed in Table II.

Fig. 6 compares the power net balance profile attained for scenario 1 in both operation modes and the original situation without storage. The global individual operation profile has been obtained aggregating the values corresponding to each prosumer in a similar way to which is done in (1) and (2). As a consequence of the improvement in $S C$ and $S S$, the coordinated operation accomplishes a reduction of energy imports and exports and, as a consequence, a lower amount of energy interchanged with the main grid and lower distribution losses. In this way, the use of the DERs of the system is maximized whereas the dependency on the main grid of the microgrid is decreased. Peak shaving is also performed along all hours of the day in both operation modes. However, the coordinated operation seems to be the most effective, because power values are lower in most hours, with a maximum reduction of power interchanged with the grid during one interval of $51 \%$.

Fig. 7 provides a comparative chart about global energy import, export, net balance and total interchange accumulated in the analyzed day for every scenario. Despite the different characteristics of every scenario, all of them agree that coordinated operation mode achieves savings in import and total interchange of energy with respect to individual operation. The most noteworthy case is scenario 1 . In that case, the total energy interchange is reduced by $12.63 \%$, as import is $20.60 \%$ lower and export is also reduced, but on a lower rate, because daily net balance is negative for both prosumers with individual operation and becomes positive with coordinated operation.

Table III contains a comparison between the proposed optimization strategy and peak shaving, in terms of the evaluation indices, when the system operates individually in the scenario 1 . The peak shaving strategy pursues to limit imported and exported power (up to $3 \mathrm{~kW}$ in our example), using the battery only to supply/store the difference between the imposed peak shaving bound and the generation-load mismatch in each household. With the conditions of the scenario 1, only power import needs to be limited for the prosumer 1 at the end of the day. Therefore, its battery is discharged at that hours satisfying (5) and (6), whereas the battery of the prosumer 2 stays idle along the entire day. In this situation, the gradient restriction cannot be complied, because the battery must provide a power gradient higher than $300 \mathrm{~W}$. This issue leads to a more abrupt operation of the battery of the prosumer 1 than with the optimized scheduling. However, the battery of the prosumer 2 is not cycled, but it is not efficiently used whereas it is degraded by calendar aging.

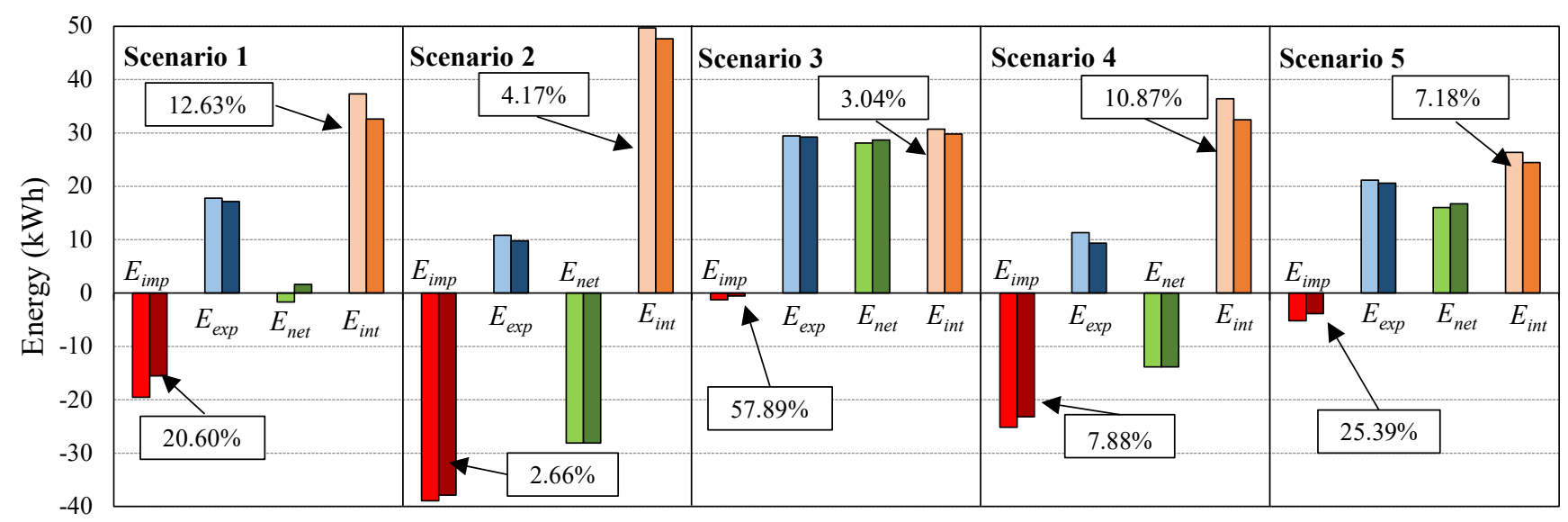

Fig. 7. Global energy import $\left(E_{i m p}\right)$, export $\left(E_{\text {exp }}\right)$, net balance $\left(E_{n e t}\right)$ and total interchange $\left(E_{\text {int }}\right)$ with the main grid for the different operations and scenarios. Light and dark colors mean individual and coordinated operations respectively. Percentages in boxes mean savings in energy imports and total interchanges between individual and coordinated operation modes in every scenario. 
TABLE III

COMPARISON OF EVALUATION INDICES FOR PEAK SHAVING AND PROPOSED OPTIMIZATION IN INDIVIDUAL OPERATION IN SCENARIO 1

\begin{tabular}{c|c|c}
\hline \hline Evaluation index & $\begin{array}{c}\text { Peak shaving- } \\
\text { scheduling }\end{array}$ & $\begin{array}{c}\text { Optimization- } \\
\text { scheduling }\end{array}$ \\
\hline$S C$ & 0.3856 & 0.5458 \\
\hline$S S$ & 0.3528 & 0.4993 \\
\hline$E_{\text {imp }}(\mathrm{kWh})$ & 52.32 & 19.49 \\
\hline$E_{\text {exp }}(\mathrm{kWh})$ & 26.10 & 17.83 \\
\hline$E_{\text {net }}(\mathrm{kWh})$ & -26.22 & -1.67 \\
\hline$E_{\text {int }}(\mathrm{kWh})$ & 39.83 & 37.32 \\
\hline \hline
\end{tabular}

Moreover, evaluation indices are worse with peak shaving objectives than with the optimized scheduling. A coordinated approach of the peak shaving scheduling could limit the power interchanged with the grid by both prosumers together up to 6 $\mathrm{kW}$ (in absolute value). Nevertheless, this situation does not happen in scenario 1 , so it would not be applied. Then, the batteries would not work and the installed capacity would be wasted without any energy management.

\section{CONCLUSION}

This work presents a novel method for scheduling the charge and discharge of batteries in an optimal way based on the minimization of the power in the point of interconnection along the entire day instead of an economic target. This approach allows obtaining solutions which are not dependent on prices or energy policies of a certain country but accomplishes inherently a set of technical, economic and environmental advantages. Moreover, this optimal scheduling has been applied to a group of prosumers in different scenarios for evaluating if their aggregation to constitute microgrids provides higher benefits than their individual operation. Results show that microgrids of prosumers working in a coordinated way comply more efficiently with the proposed objective function, with interesting savings regarding energy interchanged with the main grid and, thus, lower distribution losses, higher self-sufficiency and better use of local DERs. Real-time control of DERs and storage systems in the microgrid primary control and demand side management are left to future works.

\section{REFERENCES}

[1] R. Zafar, A. Mahmood, S. Razzaq, W. Ali, U. Naeem, and K. Shehzad, "Prosumer based energy management and sharing in smart grid," Renewable and Sustainable Energy Reviews, vol. 82, no. 1, pp. 16751684, Feb. 2018. DOI: 10.1016/j.rser.2017.07.018

[2] (2017). The Strategic Energy Technology (SET) Plan. At the heart of Energy Research \& Innovation in Europe. European Commission. [Online] Available: https://setis.ec.europa.eu/sites/default/files/ setis\%20reports/2017_set_plan_progress_report_0.pdf.

[3] S. Bellekom, M. Arentsen, and K. van Gorkum, "Prosumption and the distribution and supply of electricity," Energy. Sustain. Soc., vol. 6, no. 1, pp. 1-17, Aug. 2016. DOI: 10.1186/s13705-016-0087-7.

[4] D. E. Olivares, A. Mehrizi-Sani, A. H. Etemadi, C. A. Cañizares, R. Iravani, M. Kazerani, A. H. Hajimiragha, O. Gomis-Bellmunt, M. Saeedifard, R. Palma-Behnke, G. A. Jiménez-Estévez, and N. D. Hatziargyriou, "Trends in microgrid control," IEEE Trans. Smart Grid, vol. 5, no. 4, pp. 1905-1919, Jul. 2014. DOI: 10.1109/TSG.2013.2295514.

[5] A. S. Vijay, S. Doolla, and M. C. Chandorkar, "Real-time testing approaches for microgrids," IEEE J. Emerg. Sel. Top. Power Electron., vol. 5, no. 3, pp. 1356-1376, Sept. 2017. DOI: 10.1109/ JESTPE.2017.2695486.

[6] (2018). Residential self-consumption of solar PV power. Schneider Electric. [Online] Available: https://solar.schneiderelectric.com/solution/residential-self-consumption/

[7] O. Jogunola, A. Ikpehai, K. Anoh, B. Adebisi, M. Hammoudeh, S.Y. Son, and G. Harris, "State-of-the-art and prospects for peer-to-peer transaction-based energy system," Energies, vol. 10, no. 12, pp. 21062133, Dec. 2017. DOI: 10.3390/en10122106.

[8] N. Liu, X. Yu, C. Wang, C. Li, L. Ma, and J. Lei, "Energy-sharing model with price-based demand response for microgrids of peer-topeer prosumers," IEEE Trans. Power Syst., vol. 32, no. 5, pp. 35693583, Sep. 2017. DOI: 10.1109/TPWRS.2017.2649558.

[9] D. J. Vergados, I. Mamounakis, P. Makris, and E. Varvarigos, "Prosumer clustering into virtual microgrids for cost reduction in renewable energy trading markets," Sustain. Energy, Grids Networks, vol. 7, pp. 90-103, Sep. 2016. DOI: 10.1016/j.segan.2016.06.002.

[10] E. Mengelkamp, J. Gärttner, K. Rock, S. Kessler, L. Orsini, and C. Weinhardt, "Designing microgrid energy markets: A case study: The Brooklyn Microgrid," Appl. Energy, vol. 210, pp. 870-880, Jan. 2018. DOI: 10.1016/j.apenergy.2017.06.054.

[11] F. A. Mohamed and H. N. Koivo, "Online management genetic algorithms of microgrid for residential application," Energy Convers. Manag., vol. 64, pp. 562-568, 2012. DOI: 10.1016/j.enconman. 2012.06.010.

[12] A. Mahesh and K. S. Sandhu, "A genetic algorithm based improved optimal sizing strategy for solar-wind-battery hybrid system using energy filter algorithm," Front. Energy, pp. 1-13, 2017. DOI: 10.1007/ s11708-017-0484-4

[13] I. Mamounakis, D. J. Vergados, and N. D. Doulamis, "A method for clustering RES prosumers towards creating target aggregated prosumption profiles based on recursive predictions," in 2017 IEEE Manchester PowerTech, Manchester, UK, Jun. 2017, pp. 1-6. DOI: 10.1109/PTC.2017.7981174.

[14] N. Mukherjee and D. De, "A new state-of-charge control derivation method for hybrid battery type integration," IEEE Trans. Energy Convers., vol. 32, no. 3, pp. 866-875, Sep. 2017. DOI: 10.1109/TEC. 2017.2695242.

[15] A. C. Luna, N. L. Diaz, M. Graells, J. C. Vasquez, and J. M. Guerrero, "Cooperative energy management for a cluster of households prosumers," IEEE Trans. Consum. Electron., vol. 62, no. 3, pp. 235242, Oct. 2016. DOI: 10.1109/TCE.2016.7613189.

[16] (2018). BMZ-Data sheet-ESS 7.0/9.0/Xl. [Online]. Available: https://www.bmzgroup.com/dokumente/Datenblatt_EnergyStorage_EN neu.pdf.

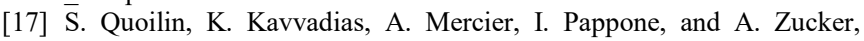
"Quantifying self-consumption linked to solar home battery systems: Statistical analysis and economic assessment," Appl. Energy, vol. 182, pp. 58-67, Nov. 2016. DOI: 10.1016/j.apenergy.2016.08.077.

[18] K. K. Zame, C. A. Brehm, A. T. Nitica, C. L. Richard, and G. D. Schweitzer, "Smart grid and energy storage: Policy recommendations," Renew. Sustain. Energy Rev., vol. 82, Part 1, pp. 1646-1654, Feb. 2018. DOI: 10.1016/j.rser.2017.07.011.

[19] S. Chouhan, D. Tiwari, H. Inan, S. Khushalani-Solanki, and A. Feliachi, "DER optimization to determine optimum BESS charge/discharge schedule using Linear Programming," in 2016 IEEE Power and Energy Society General Meeting (PESGM), Boston, MA, 2016, pp. 1-5. DOI: 10.1109/PESGM.2016.7741576.

[20] R. K. Bonthu, H. Pham, R. P. Aguilera, and Q. P. Ha, "Minimization of building energy cost by optimally managing PV and battery energy storage systems," in 2017 20th Int. Conf. Electrical Machines and Systems (ICEMS), Sydney, NSW, 2017, pp. 1-6. DOI: 10.1109/ICEMS.2017.8056442.

[21] (2015). Best practices on renewable energy self-consumption. Commission Staff Working Document. European Commission. [Online] Available: https://ec.europa.eu/energy/sites/ener/files/ documents/1_EN_autre_document_travail_service_part1_v6.pdf

[22] Y. Baghzouz, "Charge/discharge control of battery energy storage system for peak shaving," in Electr. Energy Storage Appl. and Technol. Conf., 2009, Seattle, Washington, USA, 2009, pp.1-6.

[23] S. Leonori, M. Paschero, A. Rizzi, and F. M. F. Mascioli, "An optimized microgrid energy management system based on FIS-MOGA paradigm," in 2017 IEEE Int. Conf. Fuzzy Systems (FUZZ-IEEE), Naples, 2017, pp. 1-6. DOI: 10.1109/FUZZ-IEEE.2017.8015438. 
[24] A. Zeh, M. Müller, M. Naumann, H. Hesse, A. Jossen, and R. Witzmann, "Fundamentals of using battery energy storage systems to provide primary control reserves in Germany," Batteries, vol. 2, no. 3, p. 29-49, Sep. 2016. DOI: 10.3390/batteries2030029.

[25] B. Weißhar and W. G. Bessler, "Model-based lifetime prediction of an LFP/graphite lithium-ion battery in a stationary photovoltaic battery system,” J. Energy Storage, vol. 14, Part 1, pp. 179-191, Dec. 2017. DOI: 10.1016/j.est.2017.10.002.

[26] E. Palacios-Garcia, A. Moreno-Muñoz, I. Santiago, I. Moreno-Garcia, and M. I. Milanés-Montero, "PV hosting capacity analysis and enhancement using high resolution stochastic modeling," Energies, vol. 10, no. 10, p. 1488-1509, Sep. 2017. DOI: 10.3390/en10101488.

[27] J. Zhao, N. Liu, and J. Lei, "Co-benefit and profit sharing model for operation of neighboring industrial PV prosumers," in 2015 IEEE Innovative Smart Grid Technologies - Asia (ISGT ASIA), Bangkok, Thailand, Nov. 2015, pp. 1-6. DOI: 10.1109/ISGT-Asia.2015. 7386992 .

[28] K. Uddin, R. Gough, J. Radcliffe, J. Marco, and P. Jennings, “Technoeconomic analysis of the viability of residential photovoltaic systems using lithium-ion batteries for energy storage in the United Kingdom," Appl. Energy, vol. 206, pp. 12-21, Aug. 2017. DOI: 10.1016/ j.apenergy.2017.08.170.

[29] R. A. Lopes, J. Martins, D. Aelenei, and C. P. Lima, "A cooperative net zero energy community to improve load matching," Renew.

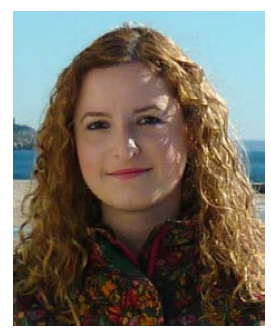

Mercedes Ruiz-Cortés received the B.Sc. degree in Electrical Engineering and the M.Sc. degree in Industrial Engineering from the University of Extremadura, Badajoz, Spain, in 2014 and 2016, respectively. She is currently pursuing the Ph.D. degree with the Power Electrical and Electronic Systems (PE\&ES) Research Group of the University of Extremadura, where she also works as a Research Assistant. Her research interests include control and management strategies of microgrids with renewable generation, energy storage systems and associated power electronic interfaces.

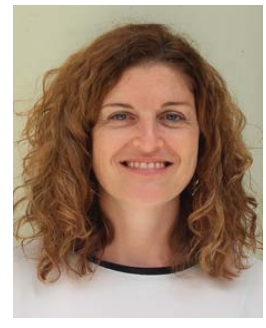

Eva González-Romera received the M.Sc degree in Industrial Engineering and the Ph.D. degree from the University of Extremadura, Badajoz, Spain, in 1998 and 2005, respectively. Currently, she is a Professor with the Electrical, Electronic and Control Engineering Department and belongs to the Power Electrical and Electronic Systems (PE\&ES) Research Group of the University of Extremadura. Her primary areas of interest are power quality, distribution grids and smart grids.

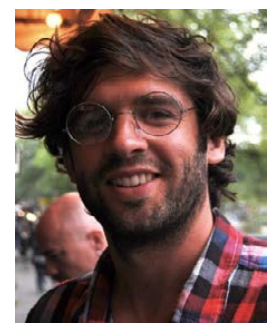

Rui Amaral Lopes received the M.Sc. and Ph.D. degrees in Electrical and Computer Engineering from the Faculty of Sciences and Technology of NOVA University of Lisbon, Caparica, Portugal, in 2012 and 2017, respectively. He is now working with the Electrical Engineering Department of this institution as Invited Assistant Professor. Additionally, since 2013 he has been with the Center of Technology and Systems-UNINOVA, Caparica, Portugal. His research is focused on energy flexibility, demand response, energy efficiency, integration of renewable energy sources, power distribution, nearly zeroenergy buildings and communities, and cooperative net-zero energy communities.

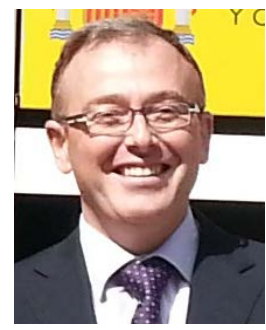

Enrique Romero-Cadaval (M'05-SM'11) received the MSc Degree in Industrial Electronic Engineering from ICAI, Comillas Pontifical University, Madrid, Spain, in 1992, and the Ph.D. degree from the University of Extremadura, Badajoz, Spain, in 2004. $\mathrm{He}$ is a Professor in Power Electronics with the Electrical, Electronic and Control Engineering Department and researcher of the Power Electrical and Electronic Systems (PE\&ES) Research Group, University of Extremadura. His research interests are
Energy, vol. 93, pp. 1-13, Aug. 2016. DOI: 10.1016/j.renene. 2016.02.044.

[30] A. R. Conn, N. I. M. Gould, and Ph. L. Toint, "A globally convergent augmented lagrangian barrier algorithm for optimization with general inequality constraints and simple bounds," Math. Comput., vol. 66, no. 217, pp. 261-288, 1997. DOI: 10.1090/S0025-5718-97-00777-1.

[31] L. Costa, I. A. C. P. Espírito Santo, and E. M. G. P. Fernandes, "A hybrid genetic pattern search augmented Lagrangian method for constrained global optimization," Appl. Math. Comput., vol. 218, no. 18, pp. 9415-9426, May 2012. DOI: 10.1016/j.amc.2012.03.025.

[32] K. Deb, and S. Srivastava, "A genetic algorithm based augmented Lagrangian method for constrained optimization," Comput. Optim. Appl., vol. 53, no. 3, pp. 869-902. Dec. 2012. DOI: 10.1007/s10589012-9468-9.

[33] M. Ceraolo, G. Lutzemberger, and D. Poli, "Aging evaluation of high power lithium cells subjected to micro-cycles," J. Energy Storage, vol. 6, pp. 116-124, May 2016. DOI: 10.1016/j.est.2016.03.006.

[34] T. Morstyn, A. Savkin, B. Hredzak, and V. Agelidis, "Multi-Agent Sliding Mode Control for State of Charge Balancing Between Battery Energy Storage Systems Distributed in a DC Microgrid," IEEE Trans. Smart Grid, vol. PP, no. 99, pp. 1-1, Feb. 2017 DOI: 10.1109/TSG.2017.2668767.

power electronics applied to power systems, power quality, active power filters, electric vehicles, smart grids, and control and integration into the grid of distributed/renewable energy resources.

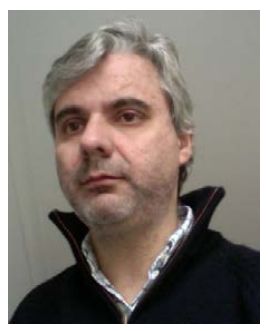

João Martins (SM'96) graduated from Electrical Engineering at Instituto Superior Técnico (IST), Technical University of Lisbon, Portugal, in 1990. $\mathrm{He}$ received his M.Sc. and Ph.D. degrees in Electrical Engineering at the same institute, respectively in 1996 and 2003. Currently he is an Associate Professor with the Department of Electrical Engineering, Faculty of Sciences and Technology, NOVA University of Lisbon, Caparica, Portugal. His research interests are energy efficiency (alternative energies and power quality, intelligent and energy efficient buildings, energy awareness, renewables integration) and advanced learning control techniques for electromechanical systems.

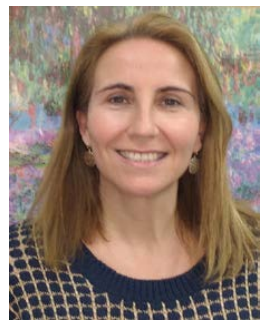

María Isabel Milanés-Montero received the M.Sc. degree in Industrial Engineering and the Ph.D. degree from the University of Extremadura, Badajoz, Spain, in 1997 and 2005, respectively. She is currently a Professor with the Electrical, Electronic and Control Engineering Department, University of Extremadura, and a member of the Power Electrical and Electronic Systems (PE\&ES) Research Group. Her major fields of expertise include power quality, renewable energy sources control, energy storage management systems, smart grids and electric vehicles.

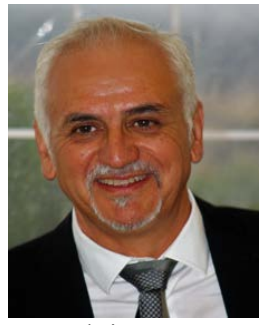

Fermín Barrero-González received the M.Sc. degree in Electrical Engineering from the Technical University of Madrid, Spain, in 1984 and the Ph.D. degree from the National Distance Education University, Madrid, Spain, in 1995. He is a Professor in Electrical Engineering with the Electrical, Electronic and Control Engineering Department, University of Extremadura, Badajoz, Spain. He is currently coordinating the Power Electrical and Electronic Systems (PE\&ES) Research Group. His research interest areas are power electronics in the power system, flexible ac transmission systems, active power filters, and electrical machine drives. 